\title{
Editorial
}

\section{Lernangebote gestalten}

Doris Lewalter

Technische Universität München
Sich verändernde curriculare Anforderungen führen u. a. dazu, dass neue, alternative Lernangebote für den schulischen Unterricht entwickelt werden. Häufig geht es darum, die Eigenaktivität der Lernenden zu erhöhen und neue, bisher unbekannte Lernerfahrungen zu ermöglichen, um den Kompetenzerwerb und v.a. auch die Lernmotivation zu unterstützen. Inwieweit mit diesen - teilweise relativ neuen - Lernangeboten die selbstgesetzten Ziele tatsächlich erreicht werden, bleibt häufig offen.

Ausgangspunkte und Zugänge für diesen Entwicklungsprozess werden für den Bereich des naturwissenschaftlichen Unterrichts exemplarisch anhand von drei Beiträgen verdeutlicht, die diese Thematik aus ganz unterschiedlichen Perspektiven aufgreifen.

Knogler und Lewalter beschreiben mit dem Design-Based-Research-Ansatz einen vielversprechenden Entwicklungszugang. Sie zeigen einen möglichen Weg auf, wie ausgehend von Praxisanforderungen wissenschaftlich und theoretisch basierte Lernangebote entwickelt und optimiert werden können und sich im selben Zug ein Beitrag zur Weiterentwicklung entsprechender theoretischer Ansätze realisieren lässt. Am Beispiel der Designentwicklung eines innovativen, motivationsfördernden Lernangebots für den naturwissenschaftlichen Unterricht wird deutlich, wie das Zusammenwirken von Praxis (Lehrkräfte) und Wissenschaft (Forscherinnen und Forscher) für beide Seiten gewinnbringend gestaltet werden kann.

Der Ausgangspunkt für die von Wecker, Rachel, Heran-Dörr, Waltner, Wiesner und Fischer vorgestellte Studie ist der Ansatz des
Forschenden Lernens. Ihm wird zwar eine förderliche Wirkung für die Entwicklung des Verständnisses für naturwissenschaftliche Inhalte zugesprochen, doch kann nicht ohne Weiteres eine Unterstützung des Erwerbs von Theoriewissen erwartet werden. Dieses Defizit bildet den Ausgangspunkt für die Arbeit der Autorengruppe. Sie zeigen, wie die Ergänzung eines am Forschenden Lernen orientierten Lernangebots um die Präsentation theoretischer Ideen dessen Effektivität für den Erwerb von Theoriewissen erhöht. Damit wird ein Weg aufgezeigt, wie durch die Weiterentwicklung bestehender Lernangebote das Erreichen grundlegender Zielsetzungen naturwissenschaftlichen Unterrichts gefördert werden kann.

Die effektive Einbindung des Museums als informelle Lernumgebung in den Unterricht stellt einen weiteren Ansatz zur Vermittlung naturwissenschaftlicher Inhalte dar, dem v. a. eine positive motivationale Wirkung zugesprochen wird. Weitgehend offen ist hierbei der Einfluss des instruktionalen Designs des Museumsbesuchs. Der Beitrag von Neubauer, Geyer und Lewalter geht der Frage nach, inwieweit typische Instruktionsformen im Museumskontext zur Unterstützung des motivationsrelevanten Erlebens der Lernenden beitragen und wie sich dessen Vorhersagekraft für das Auftreten einer inhaltsbezogenen Lernmotivation gestaltet.

Insgesamt machen alle drei Beiträge deutlich, dass es sich bei der (Weiter-)Entwicklung bestehender Lernansätze und -angebote und deren Analyse um ein wichtiges Forschungsfeld handelt, das sowohl für Theorieentwicklung als auch für die (schulische) Praxis einen wesentlichen Beitrag leisten kann. 